\title{
Sistema Kanban no gerenciamento de leitos: avaliação dos indicadores hospitalares em uma maternidade de referência
}

\author{
Kanban system in bed management: evaluation of hospital indicators in a reference maternity \\ hospital
}

Sistema Kanban en la gestión de camas: evaluación de indicadores hospitalarios en una maternidad de referencia

Recebido: 26/11/2021 | Revisado: 03/12/2021 | Aceito: 17/12/2021 | Publicado: 01/01/2022

Enewton Eneas de Carvalho ORCID: https://orcid.org/0000-0001-8545-6856 Universidade Federal do Piauí, Brasil E-mail: enewtoncarvalho@hotmail.com

Diego João de Lima Arrais ORCID: https://orcid.org/0000-0003-3399-5561 Universidade Federal do Piauí, Brasil E-mail: diegoarraissj@hotmail.com

Yara Amorim de Aguiar ORCID: https://orcid.org/0000-0002-1993-9785 Universidade Federal do Piauí, Brasil E-mail: nurse.yara@hotmail.com

Maria de Padua Martins Mendes ORCID: https://orcid.org/0000-0002-4079-8139 Universidade Federal do Piauí, Brasil E-mail: mariapadua_@hotmail.com

Lorena Citó Lopes Resende Santana ORCID: https://orcid.org/0000-0002-9968-6406 Universidade Federal do Piauí, Brasil E-mail: lorenacito@gmail.com

\begin{abstract}
Resumo
Analisar os resultados alcançados em indicadores de desempenho hospitalar com a implementação do sistema Kanban para o gerenciamento de leitos de uma maternidade de referência em gestação de alto risco. Pesquisa retrospectiva e transversal com abordagem quantitativa. Realizada em uma maternidade de ensino de Teresina - PI, referência terciária para a assistência materno-infantil. Utilizou-se dados secundários coletados no período antes e após a implementação do sistema Kanban, entre os meses de janeiro a dezembro de 2017 e os meses de janeiro a dezembro de 2018. Realizouse os testes de Qui-quadrado, Kolmogorov-Smirnov e teste não-paramétrico U de Mann-Whitney com auxílio do Software Statistical Package for the Social Sciences considerando o intervalo de confiança de 95\%. Houve um aumento de internações e altas maternas após a implementação do Kanban na maternidade em que tiveram 7122 admissões $(48,8 \%)$ e $7472(51,2 \%)$ nos anos 2017 e 2018, respectivamente. Em relação as altas houveram $5132(46,7 \%)$ e 5854 $(53,3 \%)$ nos anos estudados. No que concerne a taxa de ocupação, teve um declínio de $95,63 \%$ para $93,7 \%$ nos anos estudados. Já, o Tempo Médio de Permanência teve um decréscimo de 9,12 para 8,08 dias e o índice de rotatividade um aumento de 4,32 para 4,83\%. Utilizar os indicadores hospitalares permite que os gestores, gerentes e profissionais de saúde tenham uma visão ampla das principais dificuldades relacionadas a rotatividade de leitos e ao fluxo e ofertas dos serviços. Conclui-se que a tecnologia Kanban foi capaz de contribuir para melhorar o gerenciamento de leitos necessário entre os profissionais incentivando a cooperação proativa no fluxo de internação e melhoria da qualidade clínica na maternidade.
\end{abstract}

Palavras-chave: Gerenciamento de Leitos; Kanban; Desempenho hospitalar.

\begin{abstract}
To analyze the results achieved in hospital performance indicators with the implementation of the Kanban system for bed management in a reference maternity hospital in high-risk pregnancy. Retrospective and cross-sectional research with a quantitative approach. Carried out in a teaching maternity hospital in Teresina - PI, a tertiary reference for maternal and child care. Secondary data collected in the period before and after the implementation of the Kanban system was used, between the months of January to December 2017 and the months of January to December 2018. The tests of Chi-square, Kolmogorov-Smirnov and Mann-Whitney U non-parametric test with the aid of the Software Statistical Package for Social Sciences considering the 95\% confidence interval. There was an increase in maternal
\end{abstract}


hospitalizations and discharges after the implementation of Kanban in the maternity where there were 7122 admissions (48.8\%) and $7472(51.2 \%)$ in 2017 and 2018, respectively. Regarding discharges, there are 5132 (46.7\%) and 5854 $(53.3 \%)$ in the years studied. With regard to an occupancy rate, there was a decline from $95.63 \%$ to $93.7 \%$ in the years studied. Meanwhile, the Average Length of Stay had a decrease from 9.12 to 8.08 days and the turnover rate increased from 4.32 to $4.83 \%$. Using hospital indicators allows managers, managers and health professionals to have a broad view of the main complications related to bed turnover and the flow and supply of services. It is concluded that the Kanban technology was able to contribute to improve the management of necessary beds among professionals, encouraging proactive cooperation in the hospitalization flow and improving clinical quality in the maternity hospital.

Keywords: Bed Management; Kanban; Hospital performance.

\section{Resumen}

Analizar los resultados alcanzados en los indicadores de desempeño hospitalario con la implementación del sistema Kanban para la gestión de camas en una maternidad de referencia en embarazo de alto riesgo. Investigación retrospectiva y transversal con enfoque cuantitativo. Realizado en una maternidad docente de Teresina - PI, referente terciario para la atención maternoinfantil. Se utilizaron datos secundarios recolectados en el período anterior y posterior a la implementación del sistema Kanban, entre los meses de enero a diciembre de 2017 y los meses de enero a diciembre de 2018. Las pruebas de Chi-cuadrado, Kolmogorov-Smirnov y Mann-Whitney U prueba no paramétrica con la ayuda del software Statistical Package for the Social Sciences considerando un intervalo de confianza del 95\%. Hubo un aumento en las hospitalizaciones y egresos maternos luego de la implementación de Kanban en la maternidad, donde tuvieron 7122 ingresos $(48,8 \%)$ y $7472(51,2 \%)$ en 2017 y 2018, respectivamente. En cuanto a las altas, hubo 5.132 $(46,7 \%)$ y $5.854(53,3 \%)$ en los años estudiados. En cuanto a la tasa de ocupación, hubo un descenso del 95,63\% al $93,7 \%$ en los años estudiados. Mientras tanto, la duración media de la estancia se redujo de 9,12 a 8,08 días y la tasa de rotación aumentó de 4,32 a 4,83\%. El uso de indicadores hospitalarios permite a los gestores, gestores y profesionales sanitarios tener una visión amplia de las principales dificultades relacionadas con la rotación de camas y el flujo y oferta de servicios. Se concluye que la tecnología Kanban fue capaz de contribuir a mejorar la gestión de las camas necesarias entre los profesionales, fomentando la cooperación proactiva en el flujo de hospitalización y mejorando la calidad clínica en la maternidad.

Palabras clave: Gestión de camas; Kanban; Desempeño hospitalario.

\section{Introdução}

Dentre os problemas atuais de saúde pública no Brasil, aquele que demonstra mais claramente a falta de assistência às necessidades de saúde da população está relacionada à demanda de pacientes maior que a oferta dos serviços.

Sem dúvida, um dos problemas centrais a ser enfrentado pela Rede de Atenção às Urgências e Emergências (RUE) é a superlotação dos serviços. O aumento da demanda por serviços de urgência e emergência é um fenômeno preocupante, que tem impactado de forma contundente a gestão da clínica e a qualidade da assistência prestada nas emergências (Bittencourt; Hortale, 2009; Medeiros; Santos, 2010).

Com o intuito de diminuir essa fragilidade regulatória imposta pela dificuldade de acesso ao sistema público de saúde e de prover um espaço democrático e solidário na gestão do Sistema Único de Saúde (SUS), estados e municípios devem ter implantado em suas instituições de saúde os complexos reguladores, os quais têm a finalidade de se articular com as centrais de atenção às urgências, centrais de internação, centrais de consultas e serviços de apoio diagnóstico e terapêutico, promovendo a melhor condição de acesso a procedimentos ambulatoriais especializados, leitos hospitalares, e tecnologias de alta densidade tecnológica para a população (Aguiar, 2017).

Os complexos reguladores compõem os sistemas logísticos, dos componentes das Redes de Atenção à Saúde (RAS) que garantem uma organização racional dos fluxos e contra fluxos de informações, produtos e usuários nas Redes de Atenção à Saúde (Mendes, 2009).

O Ministério da Saúde (MS) através da Portaria nº 1.559, de agosto de 2008, instituiu a Política Nacional de Regulação do Sistema Único de Saúde, considerando a necessidade de fortalecimento dos instrumentos de gestão do SUS, que garantem a organização das redes e fluxos assistenciais, provendo acesso equânime, integral e qualificado aos serviços de saúde.

Nesse contexto, os Núcleos Internos de Regulação (NIR) das instituições de saúde definidos e recomendados pela Política Nacional de Atenção Hospitalar (PNHOSP), constituem a interface com as Centrais de Regulação para delinear o perfil 
de complexidade da assistência da instituição no âmbito da RAS do SUS, disponibilizando leitos de internação, segundo critérios pré-estabelecidos para o atendimento (Brasil, 2013).

Assim, os NIR deverão orientar a realização da gestão de leitos intra-hospitalares, possibilitando o monitoramento do paciente desde a sua chegada à instituição, durante o processo de internação e sua movimentação interna e externa, até a alta hospitalar (Brasil, 2007).

Nessa perspectiva a avaliação e incorporação de tecnologias em saúde faz parte de uma das ações da Regulação de Sistemas de Saúde efetivada pelos atos de regulamentação, controle e avaliação de sistemas de saúde, regulação da atenção à saúde e auditoria sobre sistemas e de gestão (Brasil, 2008).

Portanto, sabe-se que uma tecnologia bastante utilizada nos serviços é o sistema Kanban, uma ferramenta desenvolvida pelos japoneses, que na área da saúde, utiliza metodologias de sinalização visual, através de um painel de cores, verde, amarelo e vermelho que orientam a gestão de profissionais de saúde na melhor regulação da oferta de leitos e do tempo de permanência desse usuário na instituição hospitalar, com o objetivo de identificar possíveis falhas administrativas e assistenciais no processo de alta (Aguiar, 2017)

De acordo com Lage Júnior e Godinho Filho (2008), Kanban é uma palavra japonesa cuja tradução literal é registro, sinal visível ou placa visível. Na gestão da saúde, faz-se a adaptação desse método para a aplicação nas unidades de urgências e/ou de internação com fins de melhoria de fluxo e resolutividade de acesso dos pacientes.

Com essa ferramenta, pode-se identificar o paciente, identificar a equipe responsável, localizar o paciente, indicar o tempo de permanência, entre outros (Heisler, 2012). Nesse sentido, a importância do Kanban como ferramenta de gestão assistencial está na possibilidade de enfrentamento de um dos maiores desafios para os sistemas de saúde: adequar a capacidade de oferta às necessidades de saúde da população. Além da necessidade de diminuir o tempo de permanência associada a melhorar o cuidado e aumentar o giro do leito (Lang, 2018).

Portanto, sabe-se que a desorganização dos fluxos de assistência hospitalar às gestantes evidencia várias barreiras ao acesso ao parto, como a falta de vagas nas maternidades. Portanto, essa peregrinação da mulher tem como consequência uma verdadeira jornada em busca de uma vaga na rede pública hospitalar, situação de risco para o binômio materno-fetal, corroborando para desfechos negativos do parto e o aumento dos indicadores de mortalidade materna e neonatal.

Por isso, ao propor mudanças na gestão do trabalho assistencial, é importante utilizar indicadores capazes de demonstrar os resultados após a intervenção. Os indicadores número de internações e índice de rotatividade podem demonstrar, quando em elevação, que está sendo proporcionada maior oferta de leitos pelo hospital. O menor Tempo Médio de Permanência (TMP) dos pacientes, além de contribuir para a maior oferta de leitos, pode indicar maior capacidade resolutiva da equipe assistencial. O indicador taxa de mortalidade atua como forte balizador da qualidade assistencial, sendo identificada melhora na assistência quando seus resultados são menores (Anschau et al., 2017).

Portanto, a adaptação do Kanban para sua aplicação no contexto hospitalar é possível, viável e de grande utilidade segundo experiências de alguns autores (Moura, 1994; Lage Junior e Godinho Filho, 2008; Heisler, 2012).

Para minimizar os problemas referente a falta de leitos foi implantado no NIR a ferramenta Kanban, inserido no processo de trabalho da Instituição, centralizando o gerenciamento de leitos hospitalares e a oferta de vagas para todo hospital, influenciando no processo decisório e na melhoria dos indicadores.

O Kanban na maternidade em estudo é dividido em níveis de classificação por cores, que informam a média de dias que o paciente encontra-se no serviço. No quadro abaixo apresenta a relação entre a cor do Kanban utilizado na tecnologia de gerenciamento de leitos e o tempo médio de permanência definido da maternidade. 
Quadro 1 - Relação entre a cor do Kanban utilizado na tecnologia de gerenciamento de leitos e o tempo médio de permanência definido pela instituição.

\begin{tabular}{|c|c|}
\hline COR PADRONIZADA & MOTIVO RELACIONADO \\
\hline $\begin{array}{c}\text { VERDE } \\
\text { Cesariana até } 24 \mathrm{~h} \\
\text { Parto normal até } 12 \mathrm{~h} \\
\text { Curetagem até } 6 \mathrm{~h}\end{array}$ & Tempo de permanência aceitável, dentro do esperado \\
\hline $\begin{array}{c}\text { AMARELO } \\
\text { Cesariana entre } 24-48 \mathrm{~h} \\
\text { Parto normal entre } 12-24 \mathrm{~h} \\
\text { Curetagem entre } 6-12 \mathrm{~h}\end{array}$ & Tempo de permanência semicrítico, exige ATENÇÃO \\
\hline $\begin{array}{c}\text { VERMELHO } \\
\text { Cesariana após } 48 \mathrm{~h} \\
\text { Parto normal após } 24 \mathrm{~h} \\
\text { Curetagem após } 12 \mathrm{~h}\end{array}$ & Período crítico, longa permanência, necessita de intervenção \\
\hline
\end{tabular}

Fonte: Autores.

Por todos esses aspectos, sabe-se que a incorporação de ferramentas tecnológicas relacionada ao gerenciamento de leitos, são identificadas como recursos importantes que impactam na melhoria da gestão hospitalar. Por isso, este estudo destinouse a analisar os indicadores de desempenho hospitalar antes e após a implementação do sistema Kanban para o gerenciamento de leitos de uma maternidade de referência em gestação de alto risco no Estado do Piauí.

O presente estudo tem como objetivo analisar os resultados alcançados em indicadores de desempenho hospitalar com a implementação do sistema Kanban para o gerenciamento de leitos de uma maternidade de referência em gestação de alto risco.

Buscando descrever os resultados alcançados no indicador tempo médio de permanência, taxa de ocupação hospitalar e índice de rotatividade antes e após a implementação do sistema Kanban, além de comparar o quantitativo e a média das internações e altas hospitalares antes e após o uso do Sistema Kanban, e por fim, elaborar uma proposta de capacitação de operacionalização do Sistema Kanban na maternidade em estudo.

\section{Metodologia}

\subsection{Delineamento do estudo}

O delineamento deste estudo foi de uma pesquisa de campo retrospectiva e transversal com abordagem quantitativa em que foi analisado o impacto do sistema Kanban em indicadores de desempenho hospitalar.

A pesquisa quantitativa tem como base a mensuração de dados quantificáveis. Além disso, deve dispor da formulação de hipóteses e recursos que possam garantir a clareza dos resultados (Prandnov; Freitas, 2013).

\subsection{Local do estudo}

A pesquisa foi realizada em uma maternidade de ensino de Teresina - PI, referência terciária para a assistência maternoinfantil. A Maternidade Dona Evangelina Rosa está inserida dentro do mapa de vinculação das gestantes do estado do Piauí como unidade assistencial de referência ao parto de alto risco. Inaugurada em 15 de julho de 1976, tem passado por reformas ambientais e estruturais, sendo considerada a maior maternidade no estado e responsável por $63 \%$ dos nascimentos ocorridos na cidade de Teresina. Apresenta, em média, 1200 internações por mês das quais 900 são partos e consiste ainda em campo de estágio de 
diferentes cursos de graduação e pós-graduação da Universidade Federal do Piauí (UFPI), Universidade Estadual do Piauí e de outras faculdades privadas (Piauí, 2019).

Atualmente, a maternidade conta com 265 leitos assim distribuídos: 08 leitos de Unidade de Terapia Intensiva (UTI) Materna, 10 leitos de UTI Materna Covid, 30 leitos de UTIN, 15 leitos de UCINco, 19 leitos de Unidade de UCINca, 06 leitos no espaço NEO Covid, 06 leitos de Centro de Parto Normal (CPN), 20 leitos Casa da Gestante, 57 leitos na Ala A, 58 leitos na Ala B, 20 leitos na Ala C, 12 leitos na Ala E e 04 leitos na 04 leitos para Rede de Atenção Psicossocial (RAPS).

\subsection{População e amostra do estudo}

O estudo foi realizado com base em dados secundários coletados pelo NIR/SAME do serviço, portanto não foi necessário a definição de cálculo amostral da pesquisa.

\subsection{Coleta de dados}

Os indicadores hospitalares pesquisados foram coletados na planilha de indicadores já realizada mensalmente pela equipe do NIR/SAME no período antes e após a implementação do sistema Kanban (entre os meses de janeiro a dezembro de 2017 e os meses de janeiro a dezembro de 2018, respectivamente).

No que concerne ao instrumento de coleta de dados, foi utilizado um formulário elaborado pelos pesquisadores com as variáveis numéricas dos indicadores de desempenho hospitalar (APÊNDICE A).

\subsection{Análise de dados}

A tabulação e distribuição dos dados foi apresentada em frequência absoluta e relativa em que o teste de Qui-quadrado foi usado para avaliar a associação entre o sistema Kanban e os indicadores de desempenho hospitalar (taxa de ocupação, tempo médio de permanência e indice de rotatividade ou giro de leito). Para a comparação das variáveis: número de internações, altas e óbitos maternas, utilizou-se o teste Kolmogorov-Smirnov para verificar a aderência à normalidade dos dados, não sendo atendido o pressuposto básico deste teste, em consequência disso fez-se o uso do teste não-paramétrico U de Mann-Whitney para as comparações das variáveis. Todos os cálculos realizados com auxílio do aplicativo estatístico, Software StatisticalPackage for the Social Sciences - (SPSS), versão 19 versão para Windows. O intervalo de confiança considerado foi de $95 \%$ e valores de $\mathrm{p}$ inferiores $\alpha=0,05$ foram considerados significativos.

\subsection{Aspectos éticos}

A execução da pesquisa foi de acordo princípios éticos da Resolução 466/12 do Conselho Nacional de Saúde (CNS) que dispõem sobre as normas regulamentadoras e diretrizes de pesquisas em saúde. Segundo esta recomendação, o projeto foi cadastrado na Plataforma Brasil e submetido à apreciação do Comitê de Ética em Pesquisa (CEP) (CAAE 37548820.9.0000.5214) da Universidade Federal do Piauí (UFPI) obtendo aprovação sob número de parecer 4343449, bem como da instituição de saúde co-participante deste estudo e os dados somente foram coletados após autorização das mesmas.

Vale ressaltar que foi disponibilizado aos responsáveis da instituição de saúde a assinatura do Termo De Compromisso de Utilização de Dados -TCUD, no qual lhes foram assegurados a confidencialidade dos dados coletados, mantendo a privacidade do seu conteúdo.

\section{Resultados e Discussão}

No final do ano de 2017, foi iniciado no Núcleo Interno de Regulação da maternidade em estudo, um projeto de gerenciamento de leitos utilizando da metodologia Kanban hospitalar com registro em um quadro, onde constavam todas as 
informações sobre o processo de admissão, internação e alta das pacientes internadas na unidade para o acompanhamento do binômio mãe e recém-nascido.

O recurso utiliza um quadro que depende essencialmente das informações fornecidas pela equipe, pois necessita do registro manual de dados importantes para que essa metodologia impactasse nos indicadores hospitalares e no processo de desospitalização das pacientes otimizando ao mesmo tempo a oferta de leitos na instituição.

A Figura 1, apresenta o modelo da ferramenta Kanban utilizado pelos enfermeiros reguladores do NIR da maternidade para registro das informações de admissão, internação e alta do binômio mãe e recém-nascidos.

Figura 1 - Quadro na Ala B da Maternidade D. Evangelina Rosa em Teresina - PI utilizando a metodologia Kanban.

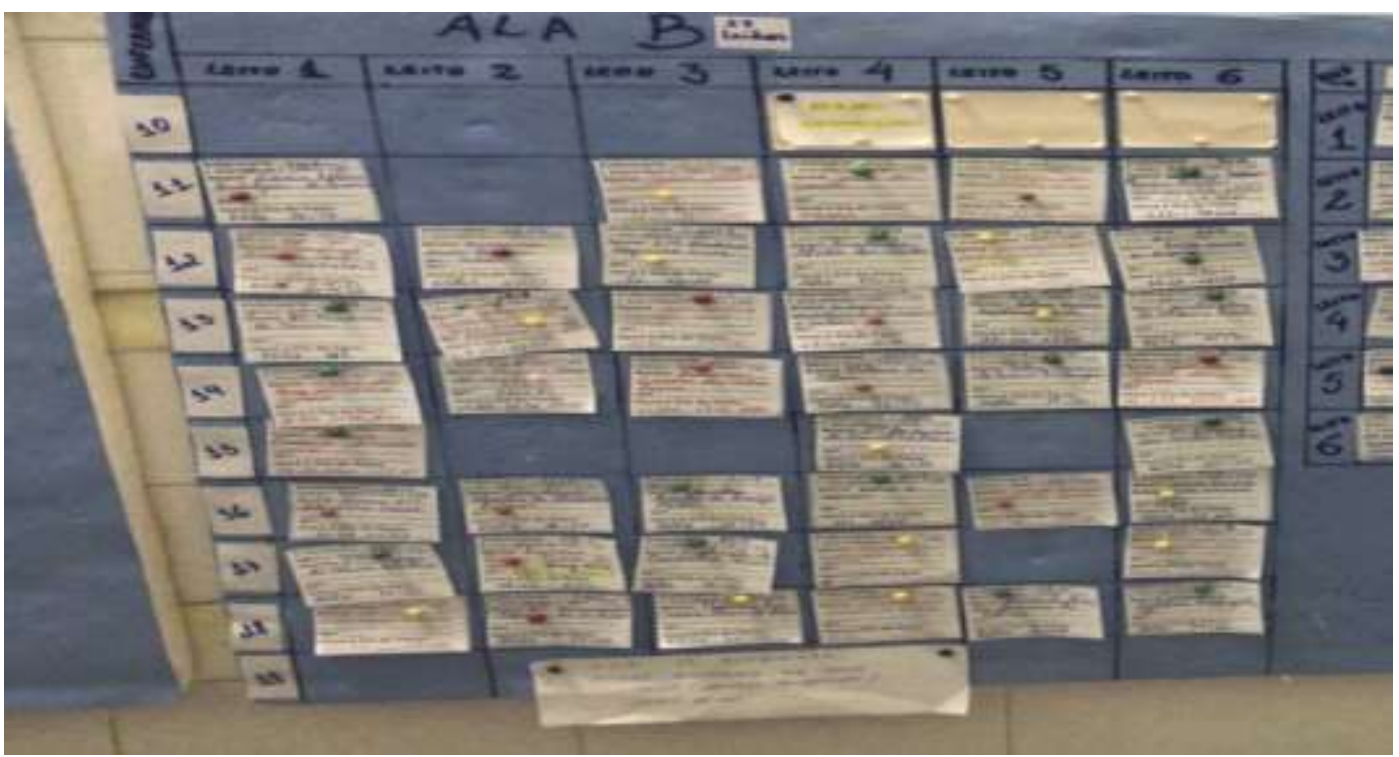

Fonte: Autores.

Os resultados apresentados abaixo constituíram na análise dos indicadores hospitalares que influenciam a regulação do acesso de pacientes a leitos obstétricos e neonatais na maternidade em estudo, ressaltando a relevância da pesquisa e a importância na implementação da referida tecnologia no ambiente hospitalar.

As variáveis de internações e altas maternas estão resumidos nas Tabelas 1 e 2 em que apresentam os quantitativos e médias nas alas A e B da maternidade dos anos de 2017 e 2018. 
Tabela 1 - Número de internações, Número de óbitos maternos e altas maternas antes e após implantação do Kanban na Maternidade D. Evangelina Rosa (Ala A e B) em Teresina - PI, nos anos de 2017 e 2018.

\begin{tabular}{|c|c|c|}
\hline \multirow{2}{*}{ Variáveis } & \multicolumn{2}{|c|}{ Período } \\
\cline { 2 - 3 } & $\mathbf{2 0 1 7}$ & $\mathbf{2 0 1 8}$ \\
\hline Internações & $7122(48,8 \%)$ & $7472(51,2 \%)$ \\
\hline Altas maternas & $5132(46,7 \%)$ & $5854(53,3 \%)$ \\
\hline
\end{tabular}

Fonte: Dados do NIR/SAME.

Na Tabela 1, os resultados demonstraram um aumento de internações e altas maternas após a implementação do Kanban na maternidade em que tiveram 7122 admissões (48,8\%) e 7472 (51,2\%), respectivamente nos anos 2017 e 2018 . Em relação as altas maternas houveram $5132(46,7 \%)$ e $5854(53,3 \%)$ nos anos estudados.

Tabela 2 - Média antes e após implantação do Kanban na Maternidade D. Evangelina Rosa (Ala A e B) em Teresina - PI, nos anos de 2017 e 2018 .

\begin{tabular}{|c|c|c|c|}
\hline \multirow{2}{*}{ Variáveis } & \multicolumn{2}{|c|}{ Período } & p-valor* \\
\cline { 2 - 4 } & $\mathbf{2 0 1 7}$ & $\mathbf{2 0 1 8}$ & \\
\hline Internações (média) & 296,75 & 311,33 & 0,409 \\
\hline Altas maternas (média) & 213,83 & 243,91 & 0,004 \\
\hline
\end{tabular}

*Teste U de Mann-Whitney: $\mathrm{p}<0.05$. Fonte: Autores.

Na Tabela 2, os resultados foram apresentados de dados coletados mensalmente descritas em médias do quantitativo de internações e altas maternas.

No ano de 2017, houveram em média 296,75 internações e 213,83 altas maternas por mês. Em relação ao ano de 2018 , houveram 311,33 internações e 243,91 altas maternas.

Esse aumento de internações e altas aconteceu em resultado similar em um estudo realizado em uma unidade de retaguarda do Hospital Nossa Senhora da Conceição, em Porto Alegre, Rio Grande do Sul onde em um período de um ano foi conclusivo para consolidar a estratégia e ratificar os resultados de qualificação assistencial e garantia maior de acesso (Anshau et al, 2017).

Além desse, segundo Petry (2016) em um estudo realizado sobre implantação do Kanban em 31 hospitais do programa SOS Emergências no Brasil um aumento nas altas hospitalares foi identificado após a implementação dessa ferramenta.

Por isso, o gerenciamento do leito hospitalar deve ser realizado levando em consideração que este é um recurso caro e complexo, o que requer que sua utilização seja feita de forma racional e com indicação apropriada, ou seja, este deve estar disponível para os indivíduos que realmente necessitem deste recurso para a recuperação de sua saúde (Ans, 2013).

Para a realização do gerenciamento de leitos nos serviços de saúde é necessário o uso de parâmetros assistenciais na adoção de indicadores hospitalares que permitam um melhor planejamento, monitoramento e avaliação das ações desenvolvidas pela instituição. Gerenciar leitos hospitalares significa utilizá-los em sua capacidade máxima dentro dos critérios definidos pela instituição, visando à diminuição da espera pela internação e evitando transferências inter hospitalares desnecessárias (Aguiar, 2017). 
Em relação a oferta de leitos hospitalares no Brasil, no ano de 2019 o país tinha 8.139 estabelecimentos hospitalares e 490.397 leitos. Isso equivale a aproximadamente 2,3 leitos para cada 1.000 habitantes, representando uma média abaixo da observada em 2017 para os países da Organização para a Cooperação e Desenvolvimento Econômico (OCDE), porém equiparada a de países como Canadá, Reino Unido e Suécia que têm sistemas de saúde majoritariamente públicos e bem organizados (Noronha et al., 2020).

Em relação ao censo hospitalar, a Secretaria de Assistência à Saúde (SAS) por meio da Portaria no 312 de 2002 padroniza a nomenclatura a ser utilizada para que as estatísticas hospitalares não sejam distorcidas e atividades como a formulação de pesquisas, interpretação das informações geradas e a realização de estudos comparativos sejam melhor executadas nos serviços existentes no país (Brasil, 2002).

A tecnologia de gerenciamento de leitos fortalece a regulação do acesso, efetivando as diretrizes de acolhimento e resolubilidade dos serviços, sendo possível garantir a suficiência de leitos obstétricos e neonatais de acordo com as necessidades da região, conforme preconiza a Rede Cegonha (Brasil, 2011).

Tabela 3 - Indicadores hospitalares antes e após implantação do Kanban na Maternidade D. Evangelina Rosa (Ala A e B) em Teresina - PI, nos anos de 2017 e 2018.

\begin{tabular}{|c|c|c|c|}
\hline \multirow{2}{*}{ Indicadores } & \multicolumn{2}{|c|}{ Período } & \multirow{2}{*}{ p-valor* } \\
\cline { 2 - 4 } & $\mathbf{2 0 1 7}$ & $\mathbf{2 0 1 8}$ & \\
\hline Taxa de ocupação & $95,63 \%$ & $93,7 \%$ & 0,072 \\
\hline Tempo Médio de Permanência & 9,12 & 8,08 & 0,586 \\
\hline Índice de rotatividade ou giro de leito & $4,32 \%$ & $4,83 \%$ & 0,185 \\
\hline
\end{tabular}

*Teste Qui-quadrado. Fonte: Autores.

$\mathrm{Na}$ Tabela 3, os indicadores demonstraram uma melhoria após a implantação do Kanban. No que concerne a taxa de ocupação, teve um declínio de 95,63\% para 93,7\% nos anos estudados. Já, o TMP teve um decréscimo de 9,12 para 8,08 dias e o índice de rotatividade um aumento de 4,32 para 4,83\%.

O resultado desse estudo corrobora com um estudo realizado em uma unidade de retaguarda do Hospital Nossa Senhora da Conceição, em Porto Alegre, Rio Grande do Sul em que no período de janeiro a dezembro de 2015 o TMP foi de 7,2 dias enquanto que no ano de 2016, após a implantação da estratégia, o TMP foi de 6,6 dias (Anschau et al, 2017).

Conforme o estudo realizado por Aguiar (2017) numa maternidade escola de Fortaleza - CE em que apresentou uma taxa de ocupação superior a 90\%, percebeu-se que a maternidade aqui de Teresina - PI possui sua ocupação dos leitos equivalente a outros locais do Nordeste.

Para Heisler (2012), a tecnologia Kanban apresentada neste estudo é "uma ferramenta de qualificação do gerenciamento do cuidado, através [...] da qual, pode-se identificar o paciente, a equipe responsável [...] indicar o tempo de permanência, dentre outros [...]."

Nesse sentido, o acompanhamento e a observação dos indicadores hospitalares apresentados, antes e após a utilização Kanban no ambiente da maternidade, tornou-se possível a avaliação da melhoria desses indicadores que mensuram a eficiência dos leitos e a qualidade dos serviços prestados pela instituição.

Ainda em relação ao gerenciamento de leitos, a PNHOSP no âmbito do SUS, instituída pela Portaria nº 3.390, de 30 de dezembro de 2013 orienta que deve ser observada a integração da prática clínica nos processos de internação e alta, com o intuito 
de aumentar a ocupação dos leitos e promover a otimização da utilização de sua capacidade, melhorando assim os atendimentos aos usuários.

Para que os gestores possam promover as ações e serviços de forma bem mais eficiente e efetiva, além de disponibilizar esses serviços com custos adequados, estes devem valer-se dos instrumentos e ferramentas da regulação, pois estas ferramentas são capazes de melhorar a ampliar dos resultados do setor (Aguiar, 2017).

Autores como Kogien (2014) e Petry (2016) apresentam o uso de tecnologias que utilizam o método Kanban como ferramenta para gerenciar leitos por meio do monitoramento do tempo médio de permanência da paciente no leito. Observa-se por meio dos estudos que este recurso facilita o processo de trabalho em equipe, permitindo uma direta, sistemática e descentralizada das vagas ofertadas e da demanda existente na instituição.

$\mathrm{Na}$ instituição onde a pesquisa foi realizada, o registro das informações de admissão, internação e alta do binômio mãe e recém-nascidos realizado pelo NIR é feito em um quadro, de forma manual e utiliza a ferramenta Kanban. Porém, as dificuldades relacionadas a atualização das informações manualmente no quadro faz repensar a construção de um sistema informatizado de gerenciamento de leitos pelo método Kanban, com atualização periódica, de fácil acesso e manuseio.

Observa-se o interesse no desenvolvimento de pesquisas sobre novos indicadores, ferramentas gerenciais e tecnologias administrativas na área da saúde. É crescente a busca por instrumentos capazes de auxiliar a gestão, medir resultados e determinar os parâmetros a seres reconhecidos e utilizados, para que sejam possíveis as comparações e juízos de valor e assim determinar o que está sendo avaliado e o que é considerado ideal (Petry, 2016).

Com base na utilização da tecnologia Kanban, os gestores hospitalares podem realizar facilmente a coleta de indicadores e com isso, gerar informações relevantes para a própria instituição e para a RAS no qual está inserida. Por meio do histórico desses indicadores e da identificação de recorrência de pontos críticos é possível gerar soluções estruturantes para a instituição. Esta análise de indicadores impactam diretamente na gestão e no cuidado, pois proporcionam resolutividade e melhoria na experiência dos pacientes (Lang, 2018).

É importante destacar que para que a tecnologia Kanban seja realmente eficaz, faz-se necessário que seu manejo seja realizado por uma equipe que tenha excelência no cuidado. Caso contrário, o emprego desta ferramenta não fará sentido e será apenas um sinalizador visual, inconsistente e burocrático (Massaro; Massaro, 2017).

Para as instituições de saúde é importante o uso de ferramentas que sejam capazes de medir os indicadores hospitalares, pois estes são recursos estatísticos que permitem aos gestores manterem controle sobre a eficácia da gestão. Outro ponto importante é que, as instituições frequentemente utilizam esses indicadores para a tomada de decisões pela gestão (Aguiar, 2017).

Nos Gráficos 1, 2 e 3 abaixo estão descritos os indicadores ao longo dos anos de 2017 e 2018 em que são observados mensalmente o tempo médio de permanência, taxa de ocupação e índice de rotatividade. 
Gráfico 1 - Tempo médio de permanência em dias antes e após implantação do Kanban na Maternidade D. Evangelina Rosa (Ala A e B) em Teresina - PI, nos anos de 2017 e 2018.

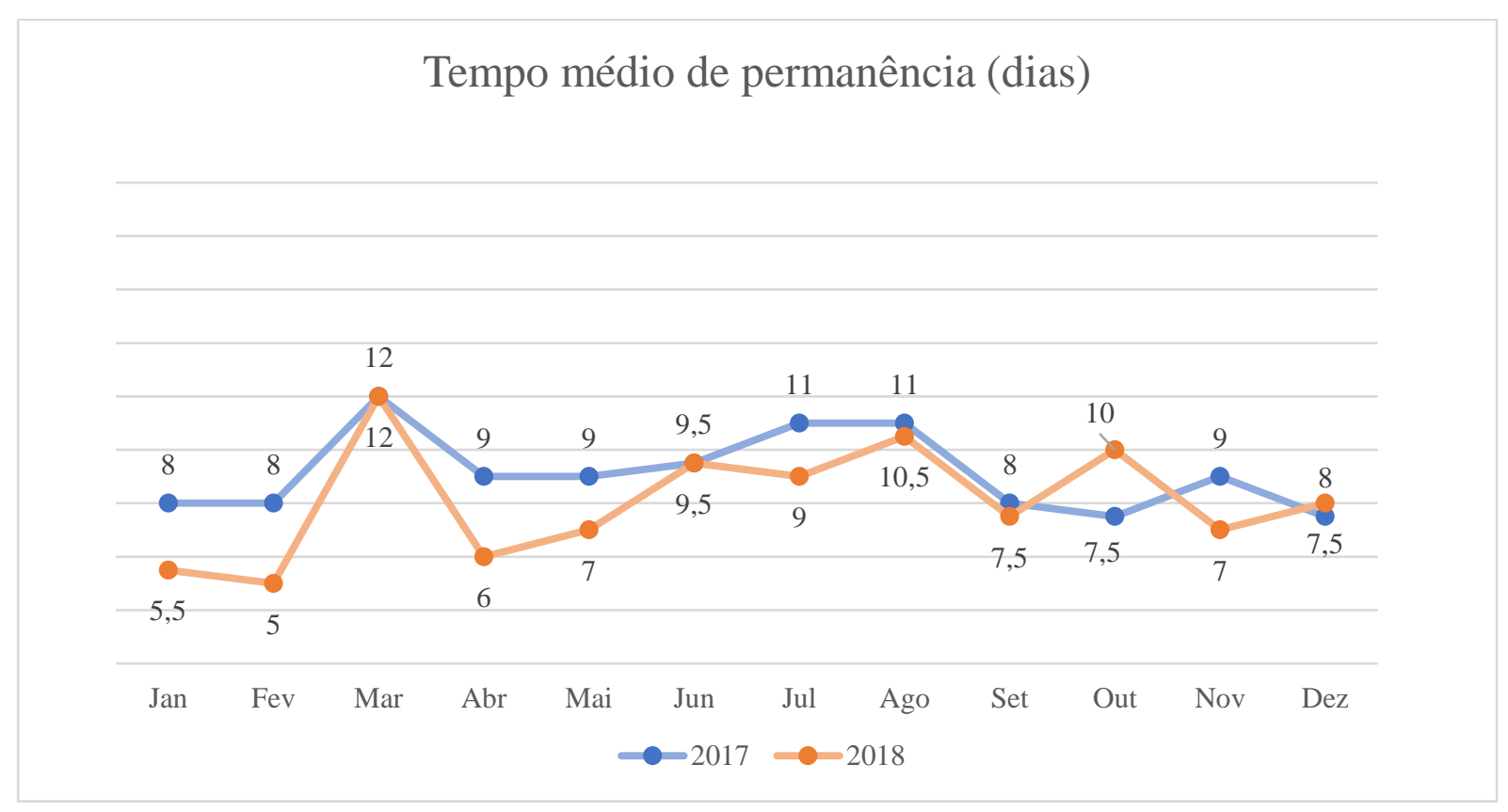

Fonte: Dados do NIR/SAME.

O Gráfico 1 apresentado analisa o TMP, observa-se variadas oscilações nesse indicador ao compararmos os dados mensalmente dos anos de 2017 e 2018, porém percebe que após a implantação do Kanban, sistema que gerencia a oferta de leitos na maternidade, esses dados vem apresentando melhorias e que no decorrer dos anos houve um decréscimo no tempo médio dos pacientes que ficaram internados na maternidade.

Sabe-se que o TMP é de extrema importância para o monitoramento da tecnologia Kanban. Este é definido como o tempo que em média um paciente permanece internado no hospital. É um indicador fortemente relacionado às boas práticas clínicas, desempenho hospitalar e também, a gestão eficiente do leito operacional.

Em um estudo realizado por Felix (2013), que implantou no Hospital Santa Marcelina - SP, um projeto denominado implantação do Método Kanban no Pronto Socorro em um Hospital Filantrópico. No relatório apresentado, a autora enfatiza que o intuito foi de melhorar a gestão do pronto socorro, por meio da melhora do fluxo e resolutividade dos pacientes. Por isso, o projeto diminuiu o tempo de permanência dos pacientes e melhorou a qualidade no atendimento e administração de recursos humanos e materiais.

Mattos (2016) em seu estudo cita também que a metodologia Kanban associada ao protocolo no fluxo de pacientes e no gerenciamento de leitos nas emergências de um hospital de Brasília, foi possível atingir os objetivos de reduzir tempo de espera, filas e tempo de internação dos pacientes, apontando assim para a eficácia desta metodologia.

Portanto, o controle do TMP pela equipe de saúde pode garantir inúmeros benefícios assistenciais para o paciente e para o hospital, o qual otimizará seus leitos com a oferta de mais vagas, aumentará a sua receita, evitando prejuízos financeiros com internações prolongadas.

Por isso, o TMP é um bom indicador de qualidade hospitalar, pois com auxílio deste, é possível medir a eficiência e a efetividade da assistência prestada ao paciente, devendo ser utilizado pelos gestores para o monitoramento. Porém, este indicador demonstra apenas se o tempo de permanência está adequado ou não de acordo com os padrões estabelecidos previamente, necessitando de outras tecnologias de gestão para que consiga identificar as causas de inadequação (Petry, 2016). 
Gráfico 2 - Taxa de ocupação antes e após implantação do Kanban na Maternidade D. Evangelina Rosa (Ala A e B) em Teresina - PI, nos anos de 2017 e 2018.

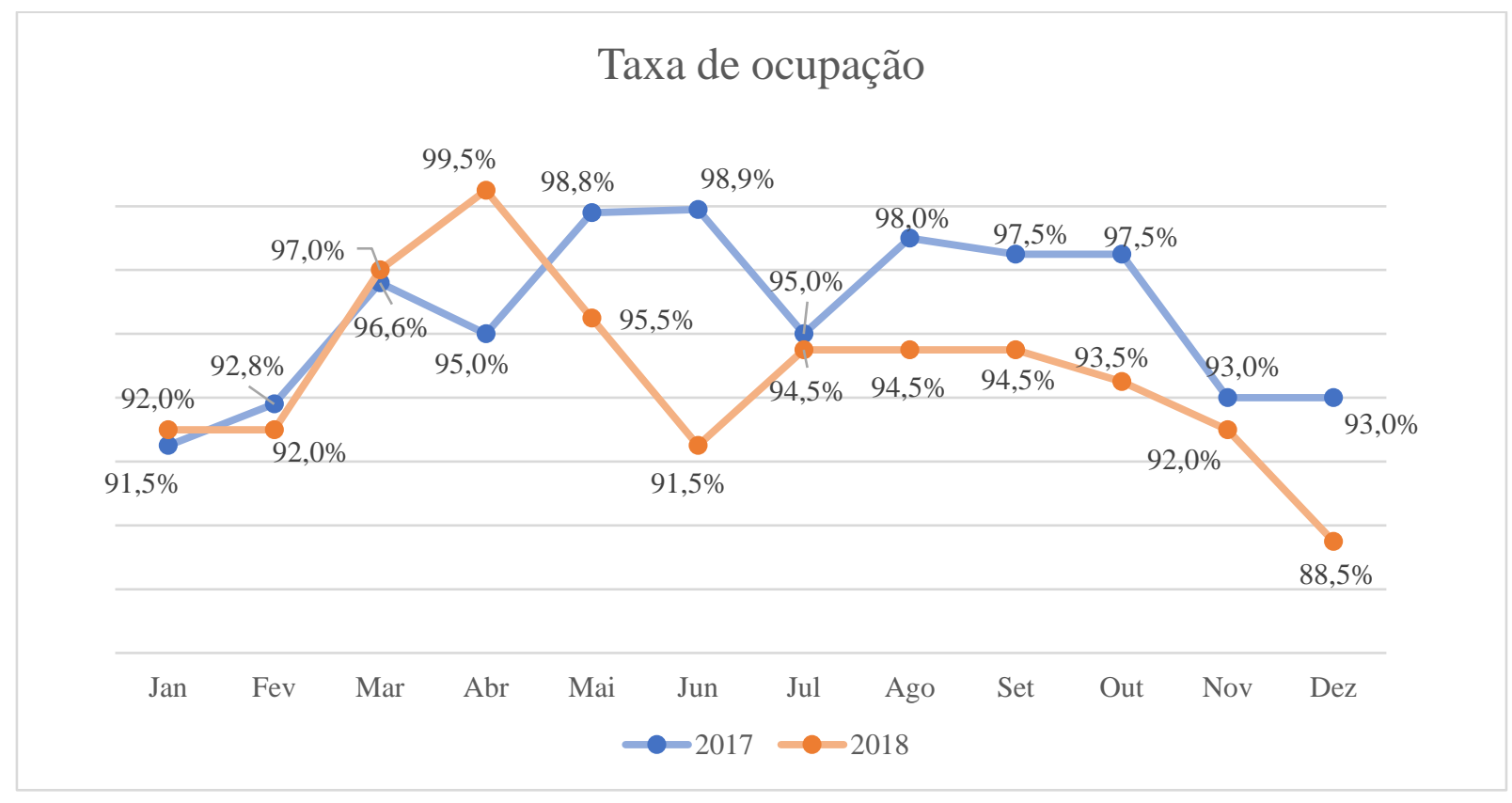

Fonte: Dados do NIR/SAME.

O Gráfico 2 apresenta a análise da taxa de ocupação hospitalar nos anos de 2017 e 2018, percebe-se no indicador uma queda nos valores quando comparados entre si e que finalizou os anos com 93\% e 88,5\% de ocupação dos leitos na maternidade, respectivamente.

Dentro do contexto de gerenciamento de leitos, a Taxa de Ocupação Hospitalar também é um indicador que precisa ser analisado. Este é definido como a relação percentual entre o número de pacientes e o número de leitos por dia, incluindo leitos bloqueados e excluindo os leitos extras existentes (Brasil, 2002).

O MS adota como parâmetro ideal uma taxa de ocupação hospitalar que varia entre um percentual de $80 \%$ a $85 \%$. Esse indicador nunca se mantém na meta recomendada pois existe um aumento no número de pacientes que buscam o serviço, o que aumenta esses valores percentuais.

Para a maternidade em que o estudo foi realizado, observa-se a mesma realidade vivenciada pelas demais instituições. Nesta, o comportamento da taxa de ocupação hospitalar é em uma média superior a 90\%, demonstrando que apesar da variação dos valores recomendados, na realidade a média é sempre maior, pois há uma elevada demanda de usuárias que buscam esta maternidade como primeira assistência materno-infantil na rede de saúde local.

Por todos esses aspectos, a superlotação traz várias consequências negativas e desgastantes para a gestão em que estão à procura de novas oportunidades para melhorar a eficiência dos processos de trabalho, através de uma assistência de qualidade que promova a satisfação do paciente.

Em suma, a maternidade em estudo é uma das principais portas de entrada de usuários, e é a unidade hospitalar que apresenta o maior número de partos realizados na capital do Estado do Piaú, sendo a única maternidade de média e alta complexidade do estado.

Portanto, utilizar os indicadores hospitalares permite que os gestores, gerentes e profissionais de saúde tenham uma visão ampla das principais dificuldades relacionadas a rotatividade de leitos e ao fluxo e ofertas dos serviços. Isso permite que os serviços tenham uma melhor facilidade nos processos de internação e alta dos pacientes (Aguiar, 2017). 
Gráfico 3 - Índice de rotatividade antes e após implantação do Kanban na Maternidade D. Evangelina Rosa (Ala A e B) em Teresina - PI, nos anos de 2017 e 2018.

\section{Índice de rotatividade}

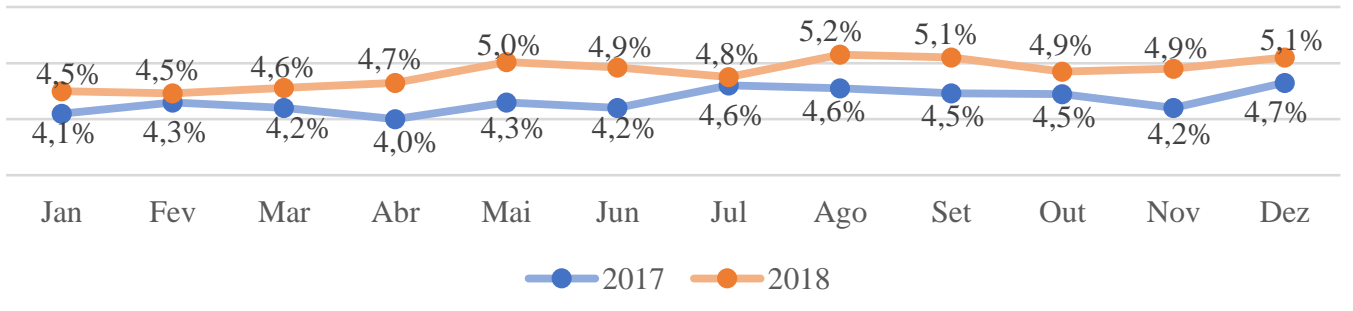

Fonte: Dados do NIR/SAME.

Por fim, no Gráfico 3 observa-se que o índice de rotatividade ou giro de leitos quando visto ao longo dos meses dos anos 2017 e 2018 existe um aumento nos valores e consequentemente representa uma melhor utilização do leito na maternidade durante o período considerado.

Um indicador bastante importante, o índice de rotatividade ou giro de leitos mostra a eficiência do uso da capacidade instalada, indicando quantos pacientes utilizaram o mesmo leito no período de um mês. Por isso, valores abaixo do esperado de giro de leitos indicam uso ineficiente do leito e paralelamente aumento do Tempo Médio de Permanência (Brasil, 2017).

No estudo realizado na maternidade como já foi falado houve um aumento no giro de leitos e corrobora com estudo realizado por Anschau (2017) em que teve resultados similares. Nele as equipes trabalhando em conjunto e utilizando as ferramentas da Gestão da Clínica, possibilitaram a redução do TMP e o aumento aproximado de 10,2\% no número de internações, com elevação do índice de rotatividade. Portanto, conforme o mesmo sabe-se que a menor duração das internações tem o potencial de diminuir a ocorrência de infecções hospitalares, aumentar o acesso da população ao sistema de saúde e reduzir os gastos assistenciais.

Os indicadores aqui expostos e as informações destes devem ser utilizados pelos gestores em saúde para determinar a adequação e a eficácia das práticas, e assim, tornar possível uma avaliação crítica do desempenho da instituição e realizar comparações com instituições similares (Programa, 2009).

Portanto, é imprescindível planejar estrategicamente a capacidade de internação da unidade de saúde. Por isso, iniciativas dedicadas a melhorar o fluxo de internação envolve projetos para reduzir o tempo de permanência e a meta é ter a todo momento uma margem de segurança em leitos disponíveis, e assim aliviar estrangulamentos de capacidade de leitos.

\section{Considerações Finais}

Conclui-se que o estudo atingiu o objetivo proposto em que a tecnologia implementada foi capaz de contribuir para aumentar os valores do índice de rotatividade ou giro de leitos, consequentemente acarretando uma diminuição do Tempo Médio de Permanência e uma redução da ocupação dos leitos da maternidade. 
Sabemos que a proposta atual de muitos governos é reduzir os gastos, expandir o acesso e melhorar a qualidade da assistência prestada. Portanto, o tempo de permanência é uma maneira eficaz de otimizar leitos garantindo a ampliação do acesso, reduzindo custos adicionais devido a superlotação.

Algumas limitações devem ser apontadas para este estudo que podem permitir melhorias para os estudos futuros. Por isso é que a proposta da tecnologia Kanban utilizada na maternidade em estudo seja cada mais informatizada, assim oferecendo uma maior cobertura de acesso materno-infantil para a população e trazendo para a equipe do NIR um recurso tecnológico mais rápido e acessível aos profissionais de Saúde de todo a maternidade, dinamizando o processo de trabalho na busca por vagas e melhor gestão dos leitos da instituição.

Diante dos dados apresentados, torna-se imperioso uma política de divulgação e capacitação plena de todos os profissionais da instituição, objetivando o pleno aproveitamento de suas potencialidades gerando como consequência, menor custo, aumento do giro do leito, maior eficiência e eficácia no cuidado ao paciente.

\section{Referências}

Anschau, F., Webster, J., Roessler, N., de Oliverira Fernandes, E., Klafke, V., da Silva, C. P., ... \& Fossari, J. A. J. (2017). Avaliação de intervenções de Gestão da Clínica na qualificação do cuidado e na oferta de leitos em um hospital público de grande porte. Scientia Medica, $27(2), 1$.

Aguiar, A. P. O. (2017). Implementação de tecnologia para o gerenciamento de leitos em maternidade de ensino. 2017.134 f. Dissertação (Mestrado Profissional em Gestão em Saúde) - Universidade Estadual do Ceará, Fortaleza.

Bittencourt, R. J., \& Hortale, V. A. (2009). Intervenções para solucionar a superlotação nos serviços de emergência hospitalar: uma revisão sistemática. Cadernos de Saúde Pública, 25, 1439-1454.

Brasil. Lei ${ }^{\circ} 11.634$, de 27 de dezembro de 2007. (2007). Dispõe sobre o direito da gestante ao conhecimento e a vinculação à maternidade onde receberá assistência no âmbito do Sistema Único de Saúde. Diário Oficial da União, seção 1, Brasília, DF, 28 dez.

Brasil. Ministério da Saúde. (2014). Implantação das Redes de Atenção à Saúde e outras estratégias da SAS. Brasília: Ministério da Saúde.

Brasil. (2017). Ministério da Saúde. Manual de implantação e implementação: núcleo interno de regulação para Hospitais Gerais e Especializados. Brasília: Ministério da Saúde. 57 p.

Brasil. (2013) Ministério da Saúde. Portaria n. 3.390, de 30 de dezembro de 2013. Institui a Política Nacional de Atenção Hospitalar no âmbito do Sistema Único de Saúde, estabelecendo-se as diretrizes para a organização do componente hospitalar da Rede de Atenção à Saúde. Diário Oficial da União, seção 1, Brasília, DF, 30 dez.

Brasil. (2011). Ministério da Saúde. Portaria n 1.459, de 24 de junho de 2011. Institui, no âmbito do Sistema Único de Saúde - SUS - a Rede Cegonha. Diário Oficial da União, seção 1, Brasília, DF, 24 jun.

Brasil. (2008). Ministério da Saúde. Portaria no 1.559 de $1^{\circ}$ de agosto de 2008. Institui a Política Nacional de Regulação do Sistema Único de Saúde - SUS. Diário Oficial da União, Brasília, DF, 04 ago.

Brasil. (2010). Ministério da Saúde. Portaria no 4.279, de 30 de dezembro de 2010. Estabelece diretrizes para a organização da Rede de Atenção à Saúde no âmbito do Sistema Único de Saúde (SUS). Diário Oficial da União, seção 1, Brasília, DF, 30 dez.

Brasil. Ministério da Saúde. Gabinete do Ministro. Portaria nº 1.459, de 24 de junho de 2011. Institui, no âmbito do Sistema Único de Saúde - SUS - a Rede Cegonha. Diário Oficial da União, Brasília, DF, 24 jun. 2011. Seção 1.

Brasil. Ministério da Saúde. Secretaria de Assistência à Saúde. Padronização da nomenclatura do censo hospitalar. - 2.ed. revista - Brasília: Ministério da Saúde, 2002 .

Felix, C. R. (2013). Implantação do método Kanban no Pronto Socorro (SUS) em um hospital filantrópico quaternário da zona leste de São Paulo. São Paulo$S P$.

Kogien, M. (2017). Implantação da ferramenta kanban e da estratégia just-in-time adaptados para a gestão do tempo de permanência do paciente em uma unidade de terapia intensiva.

Lage Junior, M., \& Godinho Filho, M. (2008). Adaptações ao sistema kanban: revisão, classificação, análise e avaliação. Gestão \& Produção, 15, 173-188.

Lang, C. E. (2018). Modelo de gestão assistencial para a rede Ebserh baseado na cogestão, núcleo interno de regulação, Kanban e painel de indicadores. 2018. 117 f. Dissertação (Mestrado Profissional em Gestão e Inovação em Saúde) - Universidade Federal do Rio Grande do Norte, Natal.

Lima, D. P., Leite, M. T. D. S., \& Caldeira, A. P. (2015). Redes de Atenção à Saúde: a percepção dos médicos trabalhando em serviços de urgência. Saúde em Debate, 39, 65-75.

Massaro, I. A. C., \& Massaro, A. (2017). O uso do KAN BAN na gestão do cuidado: superando limites. Revista de Administração em Saúde, 17(66). 
Research, Society and Development, v. 11, n. 1, e2311123926, 2022

(CC BY 4.0) | ISSN 2525-3409 | DOI: http://dx.doi.org/10.33448/rsd-v11i1.23926

Mattos, C. M. (2016). Aplicação do Kanban ao processo de internação de uma unidade hospitalar pediátrica. Niterói. Dissertação [Mestrado Profissional em Enfermagem Assistencial] - Escola de Enfermagem Aurora de Afonso Costa, Universidade Federal Fluminense.

Medeiros, R. M. Santos, M. N. (2010). Construindo Indicadores de Qualidade Assistencial em um Serviço de urgência e Emergência. Porto Alegre: GHC, 2010. Projeto de pesquisa. Especialização em Enfermagem. HNSC, Gerência de Pacientes Externos, Grupo Hospitalar Conceição.

Mello Jorge, M. H. P. D., Gotlieb, S. L. D., Soboll, M. L. D. M. S., Baldijão, M. F. D. A., \& Latorre, M. D. R. D. D. O. (1992). Sistema de informação sobre nascidos vivos-SINASC. In Sistema de informação sobre nascidos vivos-SINASC (pp. 45-45).

Mendes, E. V. (2009). Programa Mãe Curitibana: uma rede de atenção à mulher e à criança em Curitiba, Paraná. Lima: Organização Pan-Americana da Saúde.

Moura, R. A. (1994). Kanban: a simplicidade do controle da produção. IMAM.

Petry, D. (2016) Análise de Implantação do Kanban em hospitais do programa sos emergências. 2016.152 f. Dissertação (Mestrado em Saúde Coletiva) Universidade Federal da Bahia, Palmas.

Prodanov, C. C., \& De Freitas, E. C. (2013). Metodologia do trabalho científico: métodos e técnicas da pesquisa e do trabalho acadêmico-2a Edição. Editora Feevale. 